Review

\title{
ACUPUNCTURE AND SOME COMMON DISEASES IN GENERAL PRACTICE
}

\author{
Y. Staykova-Pirovska ${ }^{1}$, L. Despotova-Toleva ${ }^{2}$ \\ ${ }^{1}$ Department Internal Medicine, Clinical Laboratory and Family Medicine, \\ Faculty of Medicine, Trakia University, Stara Zagora, Bulgaria \\ ${ }^{2}$ Medical University - Plovdiv, Plovdiv, Bulgaria
}

\begin{abstract}
INTRODUCTION: Acupuncture is a traditional Chinese medical therapy that uses thin metal needles to puncture the skin at specific points on the body to relieve pain and promote wellbeing. In this paper we provide a literature overview of scientific data and articles in different countries which shows the spread of using acupuncture for some common conditions to a general practice, including musculoskeletal complaints, depression, chronic pain, cold, insomnia and others. Furthermore, we discuss the practical issues concerning the integration of acupuncture and alternative/complementary medicine into primary care to create coordinated, patient-centered care.

MATERIALS AND METHODS: Bibliographic study of scientific reports and materials from different countries where such studies have been carried out in recent years.

RESULTS: After analyzing data we found out that acupuncture is a safe, traditional Chinese medical therapy that is often perceived as both calming and relaxing for patients. Acupuncture leads to clinically relevant short- and long-term benefits for low back pain, knee osteoarthritis, irritable bowel syndrome and other conditions, reduce pain, calming the mind in patients with depression and insomnia.

CONCLUSION AND DISCUSSION: The integration of acupuncture into a primary care appears to be cost-effective for such common conditions. Furthermore, the practice of acupuncture in primary care involves rigorous training, financial discipline, and the art of communication. When it is done correctly, acupuncture proves to be beneficial for both patients and providers.
\end{abstract}

Key words: acupuncture, general practice, alternative/complementary medicine, diseases

\section{INTRODUCTION}

Acupuncture is a traditional Chinese medical therapy that uses thin metal needles to puncture the skin at specific points on the body to relieve pain and promote wellbeing. It is part of Traditional Chinese Medicine (TCM) one of the oldest healing systems still currently utilized by a significant proportion of Chinese and world populations $(1,2)$. One of the many key concepts in TCM is "Qi," a vital energy that circulates throughout the body in 14 main channels called meridians. When the flow of Qi becomes obstructed, pain or illness occurs. Putting needles at specific acupuncture points along the meridian seeks to "open the channel" and promote the healthy flow of Qi and, with that, health is restored $(1,2,3)$. There are many factors that affects the balance of the body they are called internal and external pathological factors, for instance emotions are internal harmful factors. Different emotions damage different organs and systems for example people who often are afraid of something they are prone to illness of kidneys (4). The external harmful factors are wind, cold, heat and others (4). TCM focuses on promoting the "inner balance" of the individual within the larger external environment. Any distortion from such balance is viewed as "ill" $(1,4)$. TCM have few main branchesacupuncture, acupressure, cupping, herbal medicine and diet, exercise like qi gong and Tai Chi. The exact age of TCM is highly debated, but the most popular thinking is of 5,000 years ago $(3,4)$. The earliest source of systematic documentation of acupuncture theory is the Huang Di Nei Jing, which dates back to the Han dynasty in the $2^{\text {nd }}$ century BC (1). Acupuncture theory and practice have evolved over the years in China and have spread throw whole world. The practice of acupuncture in China, as well as in the West, has become more neuromuscular-based for some musculoskeletal and neurological 
conditions (1). There are many different ways to practice acupuncture and which method to use will depend on the practitioner's theoretical assumptions, in traditional acupuncture needles are inserted into the body in accordance with the TCM framework $(1,5)$. Neoclassical acupuncture refers to a variety of methods that have arisen over the last few decades. Trigger point acupuncture is a style where the practitioner identifies and inserts needles into tender points on the body (5). Electroacupuncture involves passing a pulsed current through the body tissues via acupuncture needles. In China electro-acupuncture is often used to treat depression (5). But in its essence acupuncture is inserting thin metal needles at acupuncture points, along the meridians, or at some tender point called also "Ashi" points.

In Chine, the needles are often manipulated until either the physician perceives the needle being grabbed by the tissues or the patient experiences "De Qi," a sensation described as a mixture of heaviness, soreness, distention, that can travel from one place to another (4-6). The session continues about 30-40 minutes, the therapist often prescribes also combination of herbs, some diet and suitable exercise for the patients case.

Many studies shows that acupuncture is safe with the most common side effects as needling pain, bruising, hematoma and dizziness $(1,7)$. Most patients perceive acupuncture as calming and relaxing. The course of acupuncture treatment typically includes 10 sessions, while many people require less and some need more $(5,7)$.

Studies shows that acupuncture is one of the most used alternative healing method in general practice $(8,9)$. Family doctors are the first contact of the patient with health system and they should know patients desire and thoughts about healing process and take it in consider. Some studies show that patients prefer to take acupuncture treatment by their family doctor (10).

In this paper we provide a literature overview of scientific data and articles in different countries which shows the spread of using acupuncture for some common conditions to a general practice, including musculoskeletal complaints, depression, chronic pain, cold, headache. Furthermore, we discuss the practical issues concerning the integration of acupuncture and alternative/complementary medicine into primary care to create coordinated, patient-centered care.

\section{MATERIALS AND METHODS}

Bibliographic study of scientific reports and materials from different countries where such studies have been carried out in recent years.

The following electronic databases were searched: MEDLINE, EMBASE, and PUBMED.

The following keywords were used: acupuncture, family medicine, primary care, common diseases, depression, headache, musculoskeletal complaints, chronic pain. We used systematic reviews and articles fool text published in recent decades.

\section{RESULTS AND DISCUSSION}

Family doctors are the first doctors which patients are looked for when there is some medical problem or other issue which patient are thinking that there doctor can solve. At most cases doctor in general practice must give the right diagnosis, try to heal the sickness, but most of all to relieve the patients pain whether it is physical or emotional, patient must leave the doctor's office calm and in good mood. It turns out that in some condition conventional medicine is not enough, patients often have complains and look for their doctor but chronic pain still disturbs them (11), according to studies chronic pain is one of most often mentioned reasons from patients to turn to acupuncture or other alternative therapy (11, 12).

Studies shows that patients use acupuncture and others therapies from CAM (complementary/alternative medicine) primarily for common problems encountered in the primary care, such as low back or neck pain, a headache, cold, anxiety, or depression and musculo-skeletal complaints.

Acupuncture and some other forms of CAM are appropriate in the early stages of disease or chronic illness, the stages most commonly treated by primary care physicians (16-18).

Most of this patients are using acupuncture, because they had lost their trust in conventional approaches, or they prefer nontraditional view of medicine (1). It is interesting that patients prefer to use acupuncture and other CAM therapies in conjunction with the conventional medical treatment. At many countries government had approved the formation of integrated hospital by implementing CAM practices that are evidence-based and proven to be safe and effective $(18,21,22)$.

Study from Germany shows that alternative therapies are used by many doctors and other 
STAYKOVA-PIROVSKA Y., et al.

therapists, the most often used are acupuncture, homeopathy, manual therapy and herbal medicines (23).

In America the distribution and use of acupuncture among specialists is lead by primary care physicians, they still continue to dominate in the physician-acupuncturist population, although anesthesiologists and pain management specialists contribute in significant proportions (1).

Acupuncture is one of the most offered and used alternative therapy at general practices in many countries like UK, Australia, US (1315). UK practices are virtually unregulated, in Germany and some Scandinavian countries have intermediate systems. Legal reforms are underway in the Netherlands and the United Kingdom (23).

A study made for 20 countries in Europe report that a wide variety of CAM is provided in private practices, especially in Austria, Switzerland, Italy, the Netherlands and Germany. The three most frequently named CAM therapies in Europe were homeopathy, acupuncture and anthroposophic medicine. Bulgaria is also presented in the study with several outpatient clinics which offer acupuncture, homeopathy, lazer therapy (20).

The use of acupuncture is widespread and patients would like their family physician to provide and supervise these therapies in the primary care setting (13).

Many researches present the use and positive effect of acupuncture in one of the most common diseases in primary care $(10,12-14$, 16-18).

Two large trials compared acupuncture to treatment of acute headaches or routine care only. With six additional trials, the authors conclude that acupuncture could be a valuable non-pharmacological tool in patients with frequent episodic or chronic tension-type headaches $(24,25)$.

Another studies detected changes in cerebral blood flow associated with pain and that acupuncture analgesia is associated with changes in the activity of the frontal lobes, brain stem, and thalami $(26,27)$.

In a reports about patients suffering from irritable bowel syndrome, authors give recommendation of using acupuncture in primary care alongside with other evidenced based treatments for long-term effect $(28,38)$.
In a study of patients with low back pain and osteoarthritis improvements in physical wellbeing were significant at 12 and 24 weeks after basic course of treatment. Weekly acupuncture was associated with short-term improvements in pain and quality of life $(10,12)$. Low back pain improved after acupuncture treatment for at least 6 months. Effectiveness of acupuncture, either verum or sham, was almost twice that of conventional therapy (33-35). The differences were still significant at long-term follow-up. Acupuncture was also significantly superior to no additional intervention $(36,37)$. Therapy with acupuncture in patients with depression were associated with significantly reduced of the symptoms at 3 months when compared to usual care alone (28). Several studies from China are reporting the use of acupuncture, especially electro-acupuncture, for treating mild and moderate depression, which was leading to calming the mind and improving the sleep $(5,39)$. Patients are reporting that acupuncture is often perceived as both calming and relaxing (7). The results of this studies indicate that the most important reason for using a CAM is for treating pain (31).

According to international cost-effectiveness threshold values, acupuncture is a costeffective treatment strategy in patients with chronic neck pain, low back pain, knee pain also and headaches $(29,30,40)$.

Studies shows that acupuncture is wanted from patients and offered by doctors, but also studied and certified in universities in many countries $(1,21)$.

After analyzing data we found out that acupuncture is a safe, traditional Chinese medical therapy that is often perceived as both calming and relaxing for patients. Acupuncture leads to clinically relevant short- and long-term benefits for low back pain, knee osteoarthritis, irritable bowel syndrome and other conditions, reduce pain, calming the mind in patients with depression and headache.

\section{CONCLUSION}

The integration of acupuncture into a primary care appears to be cost-effective for such common conditions. Furthermore, the practice of acupuncture in primary care involves rigorous training, financial discipline, and the art of communication. When it is done correctly, acupuncture proves to be beneficial for both patients and providers. 


\section{REFERENCES}

1. Mao, J. and Kapur, R., Acupuncture in primary care. Prim CareMar; 37(1):105-17, 2010.

2. Cheng, X., Chinese acupuncture and moxibustion. Foreign Languages Press, Beijing, 251-260, 1987.

3. Kaptchuk, T., Acupuncture: theory, efficacy, and practice. Ann Intern Med. 136(5):374-383, 2002.

4. Zhufan, X., Practical Traditional Chinese Medicine.Foreign languages press, Beijing, ISBN 7-119-02375-6, 7-16, 2000.

5. Trine, S. and Jianping, L., Acupuncture treatment for depression-A systematic review and meta-analysis. European Journal of Integrative Medicine, 3(4): 259270, 2011.

6. Mao, J., Farrar, J., Armstrong, K., Donahue, A., Ngo, J. and Bowman, M., De qi: Chinese acupuncture patients' experiences and beliefs regarding acupuncture needling sensation--an exploratory survey. Acupunct Med. 25(4):158-165, 2007.

7. Macpherson, H., Scullion, A., Thomas, K, and Walters, S., Patient reports of adverse events associated with acupuncture treatment: a prospective national survey. QualSaf Health Care,13(5):3493552004.

8. Zollman, C. and Vicker, A., Complementary medicine and the doctor, BMJ, 319:1558, 1999.

9. Giannelli, M., Cuttini, M., DaFre, M. and Buiatti, E.,General practitioners knowledge and practice of complementary/alternative medicine and its relantionship with lifestyles:a population-based survey in Italy. BMC Family Practice , 1-8, 2007.

10. Fixlera, M., Ogdenb, C., Moirb, F. and Polleyb, M., Patient experience of acupuncture provision in a GP practice. Complementary Therapies in Clinical Practice, 18(3):140-144, 2012.

11.Chi-Keong, Petersen, S. and Brown, S., Health Status of People Using Complementary and Alternative Medical Practitioner Services in 4 English Counties. Am J Public Health. 92(10): 1653-1656, 2002.

12. McKee, D., Kligler, B., Fletcher, J., Biryukov, F., Casalaina, W., Anderson, B. and Blank, L., Outcomes of Acupuncture for Chronic Pain in Urban. Primary Care Journal Am Board Fam Med;26(6):692700, 2013.

13. White, A., Resch, K. and Ernst, E, Complementary medicine: use and attitudes
STAYKOVA-PIROVSKA Y., et al. among GPs. Fam Pract. ;14(4):302-306, 1997.

14.Perry, R. and Dowrick, C., Complementary medicine and general practice: an urban perspective. Complement Ther Med;8(2):71-75, 2000.

15.Thomas, K., Nicholl, J. and Fall, M., Access to complementary medicine via general practice. British Journal of General Practice, 51(462), 25-30, 2001.

16. Frenkel, M., Arye, E., Carlson , C. and Sierpina, V., Integrating Complementary and Alternative Medicine Into Conventional Primary Care: The Patient Perspective. The Journal of Science and Healing , 4(3):178186, 2008.

17.Hui, F., Boyle, E., Vayda, E. and Glazier, R., A randomized controlled trial of a multifaceted integrated complementaryalternative therapy for chronic herpes zoster-related pain. Altern Med Rev, 17(1):57-68, 2012.

18. Hasan, S., Ahmed, S., Bukhari, N., Cheah, W. and Loon, W., Use of complementary and alternative medicine among patients with chronic diseases at outpatient Clinics. Complementary Therapies in Clinical Practice internationally journal , 15( 3), 152-157, 2009.

19.Wong, W., Lam, L., Li ,R., Ho, H., Fai, L.K. and Li, Z., A comparison of the effectiveness between Western medicine and Chinese medicine outpatient consultations in primary care. Complementary Therapies in Medicine, 19(5), 264-275, 2011

20.Längler, A., and Zuzak, T.J., Complementary and alternative medicine in paediatrics in daily practice-A European perspective Complement. Ther Med.,21(1), 26-33, 2013.

21.Owen, D., Lewith, G. and Stephens, C.R, Can doctors respond to patients' increasing interest in complementary and alternative medicine? BMJ, 322(7279): 154-158, 2001.

22.Barberis, L., de Toni, E., Schiavone, M., Zicca ,A. and Ghio ,R., Unconventional medicine teaching at the Universities of the European Union., 29(8):559-562, 1997.

23.Fisher, P., and Ward, A., Complementary medicine in Europe. Med J, 181(4): 222225, 2004

24.Linde, K., Allais, G., Brinkhaus ,B., Manheimer ,E., Vickers, A. and White, AR., Acupuncture for tension-type headache. Cochrane Database Syst Rev. 21;(1):CD007587, 2009.

25.Melchart, D., Weidenhammer, W., Streng, A., Hoppe, A., Pfaffenrath, V. and Linde, K., Acupuncture for chronic headaches--an 
STAYKOVA-PIROVSKA Y., et al.

epidemiological study. Headache. ;46(4):632-41, 2006.

26. Vickers, A., Rees, R., Zollman, C., McCarney, R., Smith, C., Ellis, N., Fisher, P. and Van Haselen, R, Acupuncture for chronic headache in primary care: large, pragmatic, randomized trial $B M J, 328: 744$, 2004.

27.Newberg, A., Lariccia, P., Lee, B., Farrar, J., Lee, L. and Alavi, A., Cerebral blood flow effects of pain and acupuncture: a preliminary single-photon emission computed tomography imaging study. $J$ Neuroimaging, 15(1):43-49, 2005.

28.Pherson, H., Tilbrook, H., Bland, M., Bloor, K., Brabyn, S., Cox, H., Kang'ombe1, A., Man, M., Stuardi, T., Torgerson, D., Watt, I. and Whorwell, P., Acupuncture for irritable bowel syndrome: primary care based pragmatic randomised controlled trial BMC Gastroenterology, 12: 150, 2012.

29.Burke, A., Upchurch, D., Dye, C. and Chyu, L., Acupuncture use in the United States: findings from the National Health Interview Survey. J Altern Complement Med.;12(7):639-648, 2006.

30.Han, J. S., Acupuncture and endorphins. Neurosci Lett.;361(1-3):258-261, 2004.

31.Chi-Keong, Petersen, S. and Brown, S., Health Status of People Using Complementary and Alternative Medical Practitioner Services in 4 English Counties Am J Public Health , 92(10): 1653-1656, 2002.

32.Hui, F., Boyle, E., Vayda, E. and Glazier, R., A randomized controlled trial of a multifaceted integrated complementaryalternative therapy for chronic herpes zoster-related pain. Altern Med Rev.;17(1):57-68, 2012.

33.Haake, M., Müller, H., Schade-Brittinger, C., Basler, H., Schäfer, H., Maier, C., Endres, H., Trampisch, H. and Molsberger, A., German Acupuncture Trials (GERAC) for chronic low back pain: randomized, multicenter, blinded, parallel-group trial with 3 groups. Arch Intern Med. 24;167(17):1892-1898, 2007.

34.Brinkhaus, B., Witt, C. M., Jena, S., Linde, K., Streng, A., Wagenpfeil, S., Irnich, D., Walther, H., Melchart, D. and Willich, S., Acupuncture in patients with chronic low back pain: a randomized controlled trial. Arch Intern Med ;166(4):450-457, 2006.

35.Cherkin, D., Sherman, K., Avins ,A., Erro, J., Ichikawa, L., Barlow, W., Delaney, K., Hawkes, R., Hamilton, L., Pressman, A., Khalsa P. and Deyo, R., A randomized trial comparing acupuncture, simulated acupuncture, and usual care for chronic low back pain. Arch Intern Med.;169(9):858866, 2009.

36. White, A., Foster, N., Cummings, M. and Barlas, P., Acupuncture treatment for chronic knee pain: a systematic review. Rheumatology (Oxford).46(3):384-390, 2007.

37.Witt, C., Jena, S., Brinkhaus, B., Liecker, B., Wegscheider, K., Willich,S., Acupuncture in patients with osteoarthritis of the knee or hip: a randomized, controlled trial with an additional nonrandomized arm. Arthritis Rheum; 54(11):3485-93, 2006.

38.Chen, Y., Chen, F., Chen, T., Chou, L. and Hwang, S., Patterns of traditional Chinese medicine use in patients with inflammatory bowel disease: a population study in Taiwan. Hepatogastroenterology.;55(8283):467-470, 2008.

39.Willich, S., Reinhold, T., Selim, D., Jena, S., Brinkhaus, B. and Witt, C., Costeffectiveness of acupuncture treatment in patients with chronic neck pain. Pain;125(1-2):107-113, 2006.

40.Van Haselen, R., Reiber, U., Nickel, I., Jakob, A. and Fisher, P., Providing Complementary and Alternative Medicine in primary care: the primary care workers' perspective. Complement Ther Med.;12(1):6-16, 2004. 